\title{
Flavonoids in rice, their role in health benefits
}

\begin{abstract}
Rice is a staple food for more than $60 \%$ of world population and it's the world's most consumed cereal. The rice grains contain many bioactive components as one of the major content which are the group of substances with beneficial health properties that are produced as secondary metabolites during grain filling. In rice flavonoids include tricin, luteolin, apigenin, quercetin, isorhamnetin, kaempferol and myricetin have human health benefits. This compound possesses many properties such as antioxidant, anticancer, antiinflammation, cholesterol lowering activity and also illustrates prevention of human diseases like cancer and cardiovascular diseases. Bioactive compounds and flavonoids show different mode of actions includes, direct radical scavenging activity, cell membrane interaction, interacting with the protein of signaling pathways, inhibition of cell proliferation, platelet aggregation, cholesterol oxidation, natural killer cell and macrophage activation and inhibition of angiogenesis.
\end{abstract}

Volume 4 Issue 3 - 2017

\section{Nand K Singh, Manjoo Rani, Sharmila Raj T, Alok Kumar Yadav}

Department of Biotechnology, Motilal Nehru National Institute of Technology, India

Correspondence: Nand K Singh, Department of Biotechnology, Motilal Nehru National Institute of Technology, Allahabad-2I I 004 (UP), India, Email singhnand@gmail.com

Received: December 18, 2016 | Published: May 08, 2017

Keywords: rice, flavonoids, health benefits

\section{Introduction}

Rice is one of the most produced and consumed cereals in the world it has an important role in the diet and health. Some compounds with antioxidant activity had been identified in rice such as bioactive compounds including photochemical, phenolic compounds, tocotrienols and tocopherols. Bioactive compounds are a group of substance that is beneficial to human health, its small quantities also present in food. The bioactive compounds are produced by the biosynthetic and metabolic pathways of other essential compounds required for plant growth and development. These compounds considered in plant cells stuff of biochemical branch reactions and are synthesized randomly. Still, many of these compounds hold some important functions of plants like protection, attraction or signaling. ${ }^{1}$ The rice grain is composed of three main parts such as bran, germ fraction and endosperm.

The bioactive components are more concentrated in rice bran region. Following dietary intake of rice, the metabolites present in rice grain shows human disease protective activities and beneficial effects on the body immune system. Photochemical are a group of compounds with health promoting properties that are present in rice bran. The health promoting effects of any cereal grains are attributed partly to the unique phytochemical content in the grain. The major phytochemicals include phenolic compounds, tocols (Tocotrienols and tocopherols), vitamin $\mathrm{E}$ and different types of flavonoids. The outer layer of grain has been shown to contain much higher levels of these bioactive compounds. ${ }^{2}$

The phenolic compounds are present in both free and insoluble form attached to the cell wall of the grain. Based on the structure, phenolic compounds can be grouped as mono phenols and polyphenolics. The phenolic acids, flavonoids, tannins are phenolic compounds among them flavonoids showing a higher level of antioxidant activity, anticancer properties as shown in fig 1. Although, various kinds of flavonoids and their content in rice are less than other cereals so their specific effects, mode of action and their importance necessitated for further investigation, which studied in subsequent section of the paper. $^{3}$

\section{Flavonoids in rice}

Flavonoids show some peculiar physiological potential including antioxidant, antitumor properties and anti-inflammation. Flavonoids are a one of the major class of phenolic compound that most commonly distributed in plant phenolics. The Flavonoids in rice are synthesized by phenylpropanoid metabolic pathway and it is a major phenolic compound. It has a 15-carbon skeleton that involves two aromatic rings linked by a three-carbon chain as shown in Figure $1 .{ }^{4}$ The flavonoids can classify into flavones, flavonols, flavanols (flavan3-ols), flavanonols, isoflavones and flavanones which occur as O-or $\mathrm{C}$-glycoside. There are seven types of flavonoids that usually reported in rice and tricin appears to be the major flavonoid in the bran, accounting for $77 \%$ of all seven flavonoids $(131.5 \mathrm{mg} / 100 \mathrm{~g})$. The other flavonoids present can be ordered as follows: luteolin (14\%)>apigenin $(6 \%)>$ quercetin $\quad(3 \%)>$ isorhamnetin $\quad(1 \%)>$ kaempferol $(<1 \%)>$ myricetin. ${ }^{4}$ Many flavonoids are shown to have anti-oxidative activity, free radical scavenging capacity, coronary heart syndrome avoidance and anticancer action whereas some flavonoids exhibit a potential effect on anti-human immunodeficiency virus functions. The biochemical activities of flavonoids and their metabolites depend on their chemical structure relative orientation of various moieties in the molecule. After absorption flavonoids are conjugated in the liver by course of glucuronidation, sulfation and methylation and metabolized to smaller phenolic compounds. ${ }^{5}$ Flavonoid act as antioxidants to inhibit free radical mediated cytotoxicity and also work as antiproliferative agents to inhibit tumor growth and also work as weak estrogen agonists-antagonists to modulate endogenous hormone activity. This review focuses on the effect of different types of flavonoids on health benefits. 


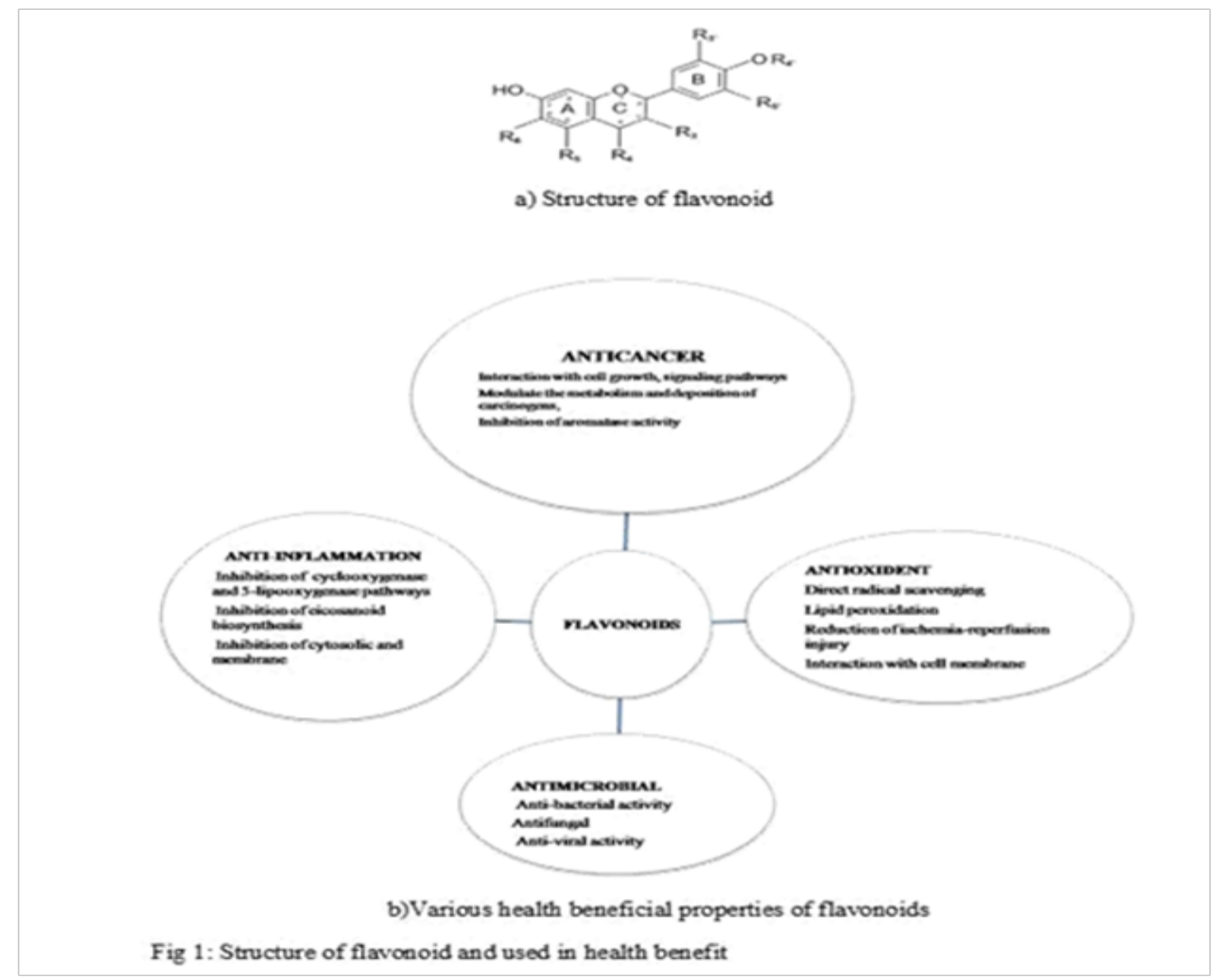

Figure I Structure of flavonoid and used in health benefit.

\section{Tricin flavonoid}

Tricin interferes with adenoma formation in the Apcmin mouse it shows in American Institute of Nutrition by providing $0.2 \%$ tricin in their diet throughout their post weaning life span up to $4-18$ weeks. Tricin consumption reduces $33 \%$ intestinal adenomas as compare to control mice. Tricin shows anticarcinogens activity in the human colon-derived epithelial cell (HCEC) and HCA-7 cell in vitro condition via inhibiting cyclooxygenase (COX) enzymes and also reduces prostaglandin E2 in the APC min mice in vivo condition. Tricin as a potential chemo preventive constituent of rice bran, study showed it interfere potently with the survival of human breast and colon cancer cells. Molecular mechanism of anti-inflammatory activity of tricin effect on NF- $\mathrm{kB}$ is signaling and pro $\square$ inflammatory genes expression using the lipo-polysaccharide (LPS) that induced human peripheral blood mononuclear cell. ${ }^{6}$ Tricin is a multifunctional nutraceutical due to the beneficial health effects such as antioxidant effect through oxidative degradation of lipid and sparing effect on vitamin $\mathrm{E}$ in erythrocyte membrane. ${ }^{?}$

\section{Apigenin flavonoid}

Apigenin was shown to inhibit the motility and invasiveness of carcinoma cells in vitro, melanoma growth and metastatic potential in vivo through administration. Apigenin flavonoid has been inhibiting cancer cell growth by different pathways such as cell cycle arrest, apoptosis in various types of human malignant tumor cells, including the breast cancer, colon cancer, prostate cancer, and leukemic cells. It also causes p21/WAF1 up-regulation of tumor suppressor p53independent manner genes in the cell cycle. Tumor

Necrosis factor-related apoptosis-inducing ligand (TRAIL) is one of the most potential candidates for cancer therapeutics. Some tumor cells are resistant to TRAIL-induced apoptosis. To overcome this resistance DR5 is a downstream gene of the p53 tumor suppressor gene. Inactivation of DR5 significantly increases tumor growth in vitro and in vivo. Therefore, the expression of DR5 may contribute to the tumor selective induction of apoptosis mediated by TRAIL apigenin up regulates DR5 expression and synergistically enhances TRAIL induced apoptosis in various types of malignant tumor cells. ${ }^{8}$

\section{Isorhamnetin flavonoid}

Isorhamnetin shows anticancer effects but molecular mechanism for the chemo preventive potential remains unknown. But it shows anti-skin cancer effects by inhibiting epidermal growth factor (EGF) 
induced neoplastic cell transformation \& also suppressed anchorage dependent and independent growth of A431 human epithelial cancerous cells. Isorhamnetin attenuated EGF-induced COX-2 expression in JB6 and A431cells, through in vivo mouse xenograft using A431cells, isorhamnetin reduced tumor growth and COX-2 expression. ${ }^{9}$

\section{Myricetin flavonoid}

Myricetin shows hypoglycemic effect studies in-vitro condition in animal. Myricetin has the function to ameliorate insulin resistance and also other functions including anti-oxidative stress, anti-inflammation, anti-aldose reductase, anti-non-enzymatic glycation and antihyperlipidemia. All of these functions may provide the contribution to the prevention of diabetic complications. ${ }^{10}$

\section{Luteolin flavonoid}

Luteolin induces apoptotic cell death in a various type of cancers via inhibits of cancer cell proliferation and suppresses tumour angiogenesis. Therefore luteolin is expected to be an anticancer therapeutic. Through in vitro results and in vivo experiments in nude mice with xenografted tumours showed that luteolin suppressed the growth of tumour formed from hepatoma, human ovarian cancer cells and human skin carcinoma, it also exerts an anti-inflammatory effect by suppressing the production of cytokines and their signal transduction pathways. Experiments with animals show that luteolin suppresses LPS and bacterial induced inflammation in vivo condition and effectively block the NF- ${ }^{\mathrm{B}}$ pathway $\&$ interfere with the function of primary (LPS) and (TNF \& IL-1) inflammatory stimulators through inhibiting IKK activation pathway through formation of the receptor signaling complex. Luteolin's shows antioxidant activity through scavenging reactive oxygen species that are responsible for oxidative stress, damage DNA, lipid and proteins and provide preventation from cancer, cardiovascular and neurodegenerative disease. ${ }^{11}$

\section{Applications of flavanoid for health benifits}

\section{Flavonoid as anti-cancer agent}

Although the main beneficial effect of flavonoid is its antioxidative property, they also have the ability to interact with different molecules along the cell growth signaling pathways and apoptosis. Reactive oxygen species (ROS) can modify the DNA and leads to a mutation in cells. If these mutations occur in oncogenes or tumor suppressor genes, the cell cycle is disturbed, and this can evolve to cancerous transformation. The metabolites of flavonoids can interact with the proteins of the signaling pathways. They alter the phosphorylation state of PI3 kinase (phosphoinositide 3kinase), Akt/PKB (proteinkinase-B), tyrosine kinase P1KC (protein-1 kinase $\mathrm{C}$ ) and mitogen activated protein (MAP) kinase of different cell signaling pathways. ${ }^{12}$ These actions of flavonoids results in the inhibition of C-Jun Kinase (JNK) and p38 pathways and the activation of extracellular signal regulated kinases (ERK), PI3-K/Akt, and Protein kinase C (PKC) pathways in a different type of cell including neuronal, cardiac, endothelial, epithelial, hepatocytes, and macrophage. ${ }^{13}$ Flavonoids interact with phase I metabolizing enzymes such as cytochrome P450 which metabolically activate a large number of procarcinogens to reactive intermediates that can interact with cellular nucleophiles and eventually trigger carcinogenesis. Flavonoid aromatase a cytochromeP450 enzyme complex synthesis and estradial that is a potent endogenous estrogen. It has been reported that estrogen involved in mammalian carcinogenesis. Aromatase inhibition can reduce growth stimulatory effects of estrogens in hormone dependent breast cancer. Some flavonoids have aromatase inhibitory property and they could be considered as potential agents against breast cancer through the inhibition of aromatase activity. Most flavonoids have been demonstrated to inhibit proliferation in numerous kinds of cultured human cancer cell lines, whereas less or no toxic to normal cells. $^{14}$

\section{Flavonoids as anti-inflammatory agent}

Another property of flavonoids is their anti-inflammation. Any inflammatory response starts with the release of arachidonic acid. Flavonoids can inhibit neutrophil degranulation by which the release of arachidonic acid by neutrophil directly blocked. They also block the cycloxygenase and lipoxygenase pathways that play an important role in the release of arachidonic acid. Another effect of flavonoids as an anti-inflammatory agent is that they inhibit biosynthesis of eicosanoid like prostaglandins that involved in various immunological responses. ${ }^{15}$

\section{Flavonoids as antimicrobial agents}

Various flavonoids are reported to show anti-microbial activities against many bacteria, viral and fungi. Some flavonoids formed as antimicrobial barriers in plants response to microbial infection. Flavanones containing cinnamoyl skeleton that exhibit inhibitory activity against Herpes simplex virus-1 (HSV-1). It observed that with an increase in glycosylation of ring-B of flavanones, inhibitory activity against PI-3 has also been increased. Flavonones with no sugar moiety showed more antibacterial activity as compare to that having sugar moiety. Flavonoids inhibit replication of human cytomegalovirus (HCMV) at the stage of viral DNA replication via the inhibition of HCMV specific DNA polymerase, and it does not inhibit immediateearly gene expression. Such types of effects of various flavonoids vary according to the compound, concentration, stimulus, and cell type. ${ }^{16}$

\section{Antioxidant activity of flavonoids}

Body cells and tissues are continuously dying out by the damage caused by free radicals. The cellular damage caused due to lipid peroxide results in cellular membrane damage. Free radicals can attract various inflammatory mediators contributing to a general inflammatory response and tissue damage. The increased production of reactive oxygen species for the period of injury results in consumption and depletion of the endogenous scavenging compounds.

Flavonoids may be a great potential to reduce various form of reactive oxygen species (ROS) through interaction with cell membranes and certain enzymes. ${ }^{17}$ Through interaction with cell membranes several non polar compounds within the hydrophobic inner membrane layer gets separated and hydrogen bonds will be formed between the polar bonds heads of lipids and the hydrophilic Flavonoids at membrane interface. Flavonoids diminish the release of peroxides and so inhibit the production of ROS by neutrophil through their interfering with the activation of $\alpha 1$-antitrypsin. ${ }^{18}$

\section{Conclusion}

Rice grain is one of the most consumed cereals worldwide. It is the source of many essential bioactive compounds such as phenolics (mono- and polyphenols), flavonoids like tricin, luteolin, apigenin; quercetin, isorhamnetin, kaempferol and myricetin have human 
health benefits. The phenolic is a part of bioactive compound present in the bran of rice grain has specific and most important health effects because they are also used to treat many diseases. This compound possesses antioxidant, anticancer, anti-inflammation, cholesterol lowering activity and prevention of human diseases like cancer, cardiovascular diseases hence flavonoids and phenolic compounds are important for human health.

\section{Acknowledgements}

None.

\section{Conflict of interest}

The author declares no conflict of interest.

\section{References}

1. Kanadaswami C, Lee L, Lee PH, et al. The antitumor activities of flavonoids. In Vivo. 2005;19(5):895-909.

2. Adil G, Wani SM, Masoodi FA, et al. Whole-grain cereal bioactive compounds and their health benefits. J Food Process Technol. 2012;3:3.

3. Bar N, Tuncel S, Yilmaz N. Gamma-oryzanol content, phenolic acid profiles and antioxidant activity of rice milling fractions. Eur Food Res Technol. 2011;233:577-585.

4. Goufo P, Trindade H. Rice antioxidants phenolic acids, flavonoids, anthocyanins, proanthocyanidins, tocopherols, tocotrienols, c-oryzanol, and phytic acid. Food Sci Nut. 2014;2(2):75-104.

5. Xiao J, Chen T, Cao H. Flavonoid glycosylation and biological benefits Biotechnol Adv. 2014;14:9.

6. Shalini V, Jayalekshmi A, Helen A. Mechanism of anti-inflammatory effect of tricin, a flavonoid isolated from Njavara rice bran in LPS induced hPBMCs and carrageenan induced rats. Mol immunol. 2015;66(2):229239.

7. Zhou J, Ibrahim RK. Tricin a potential multifunctional nutraceutical. Phyto Chem Review. 2010;9(3):413-424.
8. Escande C, Nin V, Price NL, et al. Flavonoid apigenin is an inhibitor of the NAD + ase CD38 implications for cellular NAD + metabolism, protein acetylation, and treatment of metabolic syndrome. Diabetes. 2013;62(4):1084-1093.

9. Kim JE, Lee DE, Lee KW, et al. Isorhamnetin suppresses skin cancer through direct inhibition of MEK1 and PI3-K. Cancer Prev Res. 2011;4(4):582-591.

10. Yashu Y, Guobei L, Yan X, et al. Preformulation studies of myricetin : A natural antioxidant flavonoid. Pharmazie. 2014;69(1):19-26.

11. Lin Y, Shi R, Wang X, et al. Luteolin, a flavonoid with potential for cancer prevention and therapy. Curr Cancer Drug Tar. 2008;8(7):634-646.

12. Cui J, Zhang M, Zhang Y, et al. JNK pathway: diseases and therapeutic potential. Acta Pharmacol Sin. 2007;28(5):601-608.

13. Williams RJ, Spencer JPE, Rice EC. Flavonoids antioxidants or signaling molecules. Free Radical Bio Med. 2004;36(7):838-849.

14. Vermeulen K, Van BDR, Berneman ZN. The cell cycle a review of regulation, deregulation and therapeutic targets in cancer. Cell Prolif. 2003;36(3):131-149.

15. Rathee $\mathrm{P}$, Chaudhary $\mathrm{H}$, Rathee $\mathrm{S}$, et al. Mechanism of action of flavonoids as anti-inflammatory agents: a review. Inflamm Allergy Drug Targets. 2009;8(3):229-235.

16. Orhan DD, Ozcelik B, Ozgen S, et al. Antibacterial, antifungal, and antiviral activities of some flavonoids. Microbio research. 2010;165(6):496-504.

17. Nijveldt RJ, Van Nood EL, Van Hoorn DE, et al. Flavonoids: a review of probable mechanisms of action and potential applications. Am J Clin Nutr. 2001;74(4):418-425.

18. Tarahovsky YS, Muzaharov EN, Kim YA. Rafts making and rafts breaking: how plant flavonoids may control membrane heterogeneity. Mol Cell Biochem. 2008;314(1-2):65-71. 